\title{
Social Navigation Support through Annotation-Based Group Modeling
}

\author{
Rosta Farzan $^{2}$ and Peter Brusilovsky ${ }^{1,2}$ \\ ${ }^{1}$ School of Information Sciences and ${ }^{2}$ Intelligent Systems Program \\ University of Pittsburgh, Pittsburgh PA 15260, USA \\ peterbepitt.edu, rostaecs.pitt.edu
}

\begin{abstract}
Closed corpus AH systems demonstrate what is possible to achieve with adaptive hypermedia technologies. However, they are impractical for dealing with the large volume of open corpus resources. Our Knowledge Sea project explores social navigation support, an approach for providing open corpus personalized guidance that is based on past learners' interaction with the system. The most recent stage of our project focuses on using annotations for social navigation support. We present here Knowledge Sea II, which implements annotation-based social navigation support, and report the results of several classroom studies, which have evaluated this technology.
\end{abstract}

\section{Introduction}

Day by day, the amount of information on the Internet grows, which makes the Internet an important resource in learning. However, learners are having a hard time finding what they are looking for and are very often frustrated with the search process. Adaptive navigation support techniques developed in the field of Adaptive Hypermedia [1] could be used to guide learners to the right resources at the right time. However, concept-based navigation support mechanisms used in traditional Adaptive Hypermedia (AH) systems are not suitable for the large volume of open corpus documents [2]. When searching for a mechanism to deal with the large scale of adaptive navigation support needed in open corpus hypermedia, we turned to the ideas of social navigation [5]. We have attempted to develop personalized navigation support techniques that are based on past learners' interactions with the system. We call this social navigation support (SNS). Unlike traditional adaptive navigation support, which relies on expert-provided knowledge about each resource, social navigation support relies on the collective knowledge of a large community of learners, casually gathered through many different forms of feedback.

We explored social navigation support in the context of Knowledge Sea, a project that currently focuses on helping students of introductory programming courses find relevant readings among hundreds of online tutorial pages distributed over the Web. In the first 
stage of our project, we explored the relatively straightforward "footprint" techniques suggested in early papers on social navigation $[4 ; 8]$. The idea of the "footprints" is to count how many users are passing through a link or visiting a page, in order to recommend the most popular links and pages. Combining the ideas of social navigation with the ideas of group modeling and adaptive navigation support, we have implemented a "socially adaptive" system, Knowledge Sea II [2]. This system changes the intensity of a cell's background color, to indicate how many users of the current group have visited each tutorial page and each cluster of tutorial pages: the more visits, the more intensive the color (Fig. 1). This kind of SNS helped the learners to clearly recognize the most and the least visited pages and guide their navigational choices appropriately. Our classroom study [2] demonstrated that a footprint-based SNS is able to increase the usage of open corpus resources and that the learners appreciate it immensely. At the same time, a few students pointed out that the number of visits to a page is not always a reliable measure of its relevance to their needs and asked for better relevance indicators.

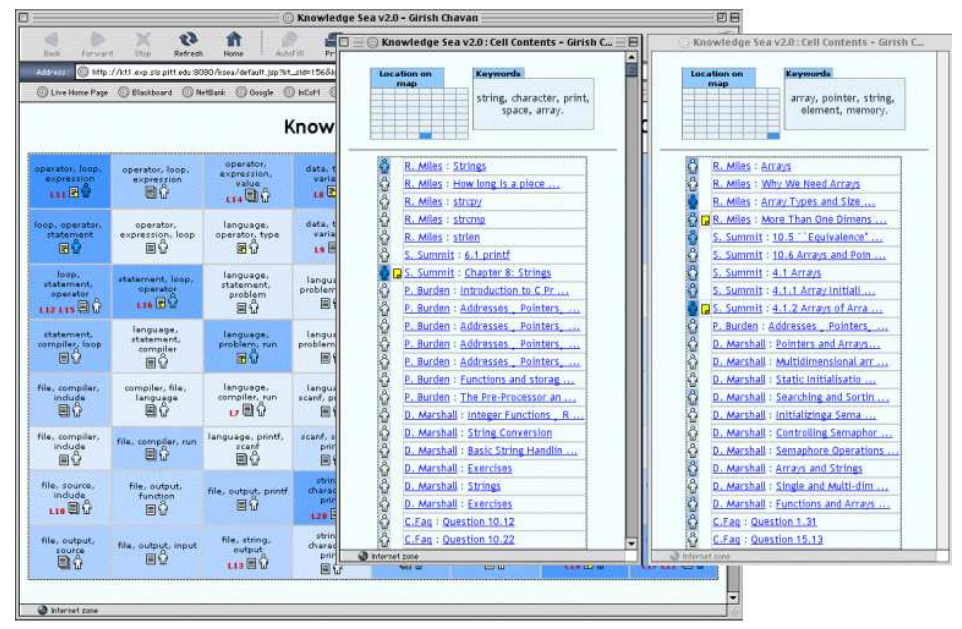

Fig. 1 - The map view and two cell views in Knowledge Sea II

This paper presents the second stage of our project, which focused on providing a more reliable SNS: predict learner interest in resources through other learners' feedback. Our main challenge was to extract feedback from actions that users are naturally performing while working with the system. To answer this challenge, we explored annotation-based social navigation support. We encouraged learners to annotate pages they are reading by writing notes or highlighting parts of the page they found important. These annotations were used as an implicit indicator of page relevance for the current group of learners. The annotation-based SNS was implemented in the newest version of Knowledge Sea II (KSII) and explored in two classroom studies. The results indicate that it is a promising approach for open corpus adaptive navigation support. In the following 
sections we introduce KSII, describe two consecutive implementations of annotationbased SNS, present the results of the classroom studies, and analyze similar projects.

\section{Annotation-based Social Navigation Support in Knowledge Sea}

The starting point for our work on annotation-based SNS was the first version of Knowledge Sea II system (Fig. 1), which combined the use of a self-organized knowledge map [3] with a simple "footprint" SNS [2]. The first version of KSII provided a simple interface for annotating tutorial pages by adding written notes. To help students navigate back to pages with notes, all such cells and pages were marked with a note icon (Fig. 1). In the first version, all notes were private: students were not able to see note icons or annotation made by others.

As a part of our evaluation of KSII, we asked the students several questions about the system's annotations. The answers showed that about $60 \%$ of the students appreciated the ability to annotate and further, were interested in sharing their annotations, seeing annotations made by others, and knowing which pages were annotated (Fig. 4). In addition, we examined the notes that the students created for themselves and discovered that almost all notes could be categorized into three groups: praise, problem, or general (37 praise, 36 problem, 34 general). This data motivated us to proceed with expanding the role of annotations in KSII and exploring the use of annotation as a source of a more reliable SNS, which we called annotation-based SNS. The current version of annotationbased SNS was developed in two phases, which were evaluated during the spring and fall semesters of 2004. The remaining part of this section presents the new features for annotation-based SNS which were introduced during these phases. The following section focuses on assessing the value of these features.

\subsection{Phase 1: Public and Private Notes}

The second version of KSII offered students the ability to make their annotations public and to choose one of three types of annotations (praise, problem, or general note). To make the presence of public annotations visible on the navigation level, we augmented the links inside the cell content window, and the links between tutorial pages with a small sticky note icon inside a yellow square. The color of the square represented the density of public annotations and the color of the sticky note represented the density of the personal annotations. Therefore, students could more easily make their navigation decisions, based on annotation information in addition to the traffic information, which had been provided in the first version of the system.

We expected two effects from the new annotation interface. We expected that the presence of public annotation would affect the students' navigational behavior, i.e., students would be more likely to visit pages with annotations. We also hoped that students 
would categorize the annotations by type, to express themselves more clearly. The main objective of the Spring 2004 classroom study was to assess these hypotheses.

\subsection{Phase 2: Stronger Annotation-Based Navigation Support}

In the second stage, we introduced several new features, motivated by the results of the Spring 2004 evaluation. From the authoring side, we attempted to encourage students to annotate by simplifying the annotation interface. To do this, we added highlighting (hypothesizing that highlighting would be easier for the students to use than writing notes). Fig. 2 presents the final version of annotation interface (available on the right side of each tutorial page). To highlight, a student can easily select part of the text inside the tutorial page and click on highlight button. Likewise, they can deselect the text. To write notes, students need to specify the following: type of the note (praise, problem, or general), visibility of the note (public versus private note), and anonymous versus signed. We added the option to sign notes in this phase in order to motivate students to share feedback with their classmates. As Fig. 2 shows, students can view any previously written notes they are the authors of but only the public notes written by others.

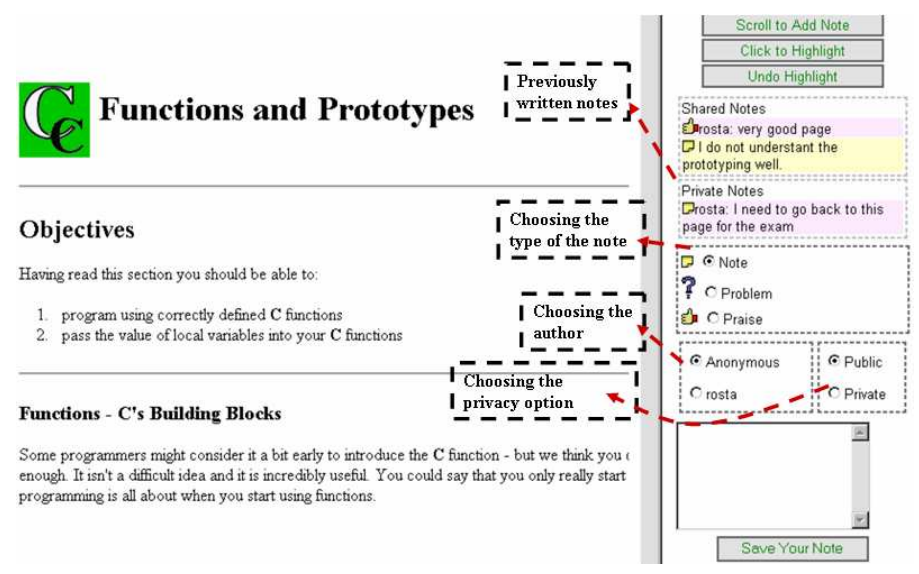

Fig. 2-Tutorial page with the annotation frame

From the usage side, we tried to offer stronger navigation support by visualizing annotation temperature and by using the annotation type, which had been provided by the student author . Every link in a cell content or a tutorial window is augmented with one or two icons inside a small square. As in the previous phase, the background color of the square gives information about the density of group annotations. The icon inside the square now indicates the type of personal annotation (if present). A thumbs-up icon indicates that the current student has written a positive annotation or has highlighted part of the page. A question mark shows that the current student has written a problem-type 
annotation, while a sticky note indicates the existence of a general note. In addition, a thermometer icon shows the "temperature" of the annotations of the students in the current group. The temperature is warmer when more students have associated positive annotations with the page and colder when more students have associated problem-type annotations with the page. Fig. 3 presents part of the cell content window with annotationbased social navigation support. Labels explain the icons in the picture below.

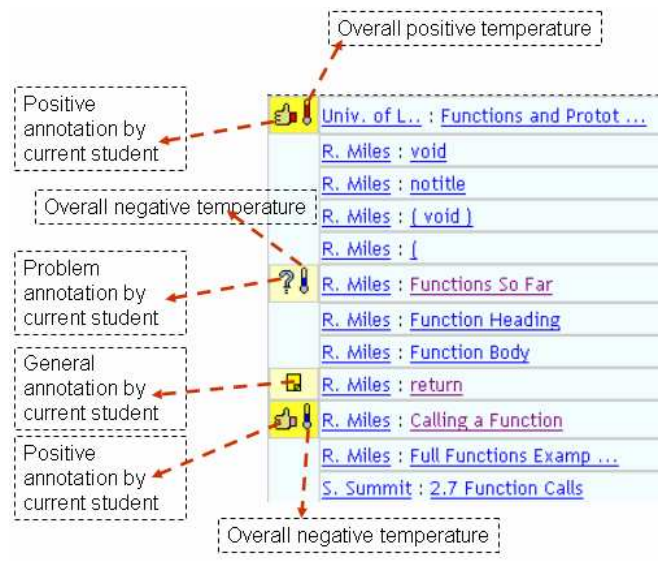

Fig. 3-Cell content window with annotation-based social navigation support

We hypothesized that the usage of annotation ability and typed notes will be higher since the benefit of it is clearer. We expected to see stronger correlation between annotated pages and students' navigation behavior. We also expected that usage of the system will increase since it is easier for students to find relevant information.

\section{Evaluation}

We assessed the effectiveness of the system and evaluated our hypotheses through a threesemester user study. The study was done in an introductory $\mathrm{C}$ programming course taught every semester at the School of Information Sciences, University of Pittsburgh. During the study, students' interaction with the system was logged. In addition, each student's opinion about the system and its features was solicited through a non-mandatory questionnaire presented at the end of each semester. Analyzing students' logs, we evaluated the effect of annotation on students' navigation and overall usage of the system. The questionnaire provided data on the students' opinion about the annotation ability of the system, their interest in visiting pages annotated by others, and their interest in sharing annotations with others. The rest of this section describes the evaluation of the system in detail. The three versions of KSII compared in this section (Fall 2003, Spring 2004, Fall 
2004) differed only in the annotation authoring and its use for navigation support, as explained in section 2 .

\subsection{Students' Attitude toward Annotations}

As shown in Fig. 4, about $60 \%$ of students appreciated the ability to annotate and very few gave negative ratings to it, within the first two semesters. This positive attitude further increased after we expanded both the authoring and usage aspects of annotations. For the most recent version, evaluated in the Fall 2004 semester, 90\% of the students found the ability to annotate to be a positive asset and none gave any negative feedback. We also asked students for their opinion of annotation-based navigation support (usage of public annotation to guide navigation). About $70 \%$ of the students rated annotation-based navigation support in a positive way. The graph shows that the enhancement of annotation abilities increased positive attitude toward annotation.

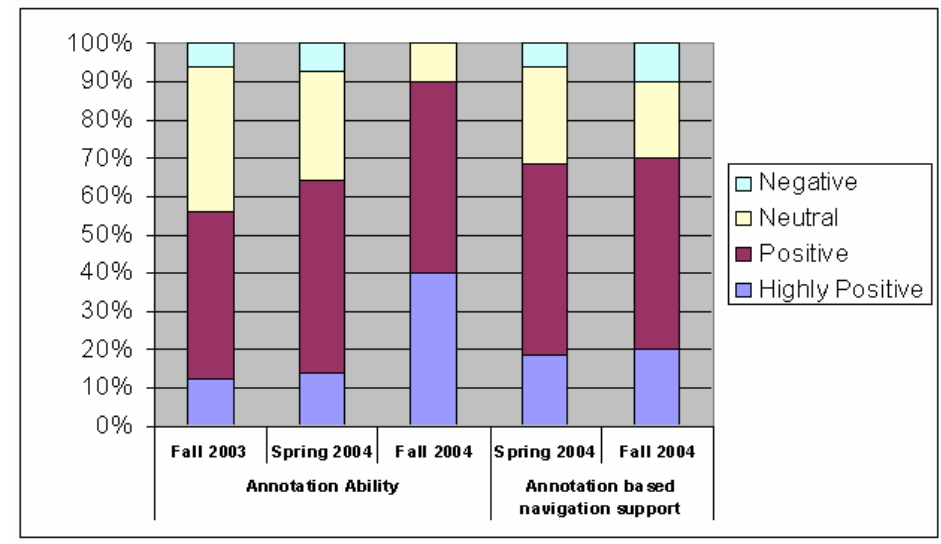

Fig. 4 - Student's opinions about the annotation feature of the system

\subsection{Effect of Annotation on Usage of the System}

Fig. 5 presents the usage of three versions of KSII over three semesters. Note that it is only in the third version of KSII, which balanced an extended annotation interface with a more comprehensive annotation-based navigation support, we were able to achieve a visible increase of all usage parameters. However, even the first simple version of annotation-based navigational support (showing only the density of public annotations) caused a very solid increase in the percentage of students actively using the system. 


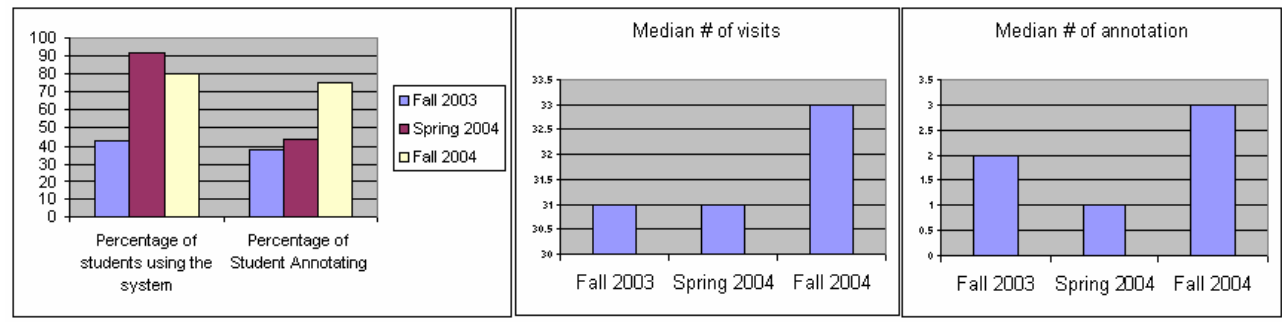

Fig. 5-Overall usage of KS system over the 3 semesters of users study

\subsection{Effect of Annotations on Students' Navigational Behavior}

To analyze the effectiveness of annotation-based navigation support, we compared navigation behavior of students related to presence of annotation. We looked at documents with public annotations over the last two semesters and analyzed the percentage of activity on each document before and after public annotation existed. We also looked at documents with public and private annotations to investigate the effect of annotation on revisiting a document by the author of the annotation.

Annotated pages versus useful pages: The first thing we have attempted to evaluate is a correlation between page quality for the given group and presence of annotation. To argue that guiding students to annotated pages is meaningful, we need to show that pages that get annotated are attractive and useful for the group. One way to evaluate the quality of a page is by the frequency of its access. Table 1 shows that page access probability (the number of visits divided by the possible number of documents to be visited) is significantly larger for annotated pages. This is a two-way correlation. From one side, most-visited pages get annotated. From the other side, the presence of annotation encourages student to visit pages.

Table 1 - Effect of annotation in visiting a page

\begin{tabular}{|l|l|l|c|c|}
\hline & \multicolumn{2}{|c|}{ Average visit } & \multicolumn{2}{c|}{ p-value } \\
\hline & Spring 2004 & Fall 2004 & Spring 2004 & Fall 2004 \\
\hline Annotated & 4.87 & 5.38 & \multirow{2}{*}{0.00001} & 0.00001 \\
\hline Not Annotated & 0.025 & 0.033 & & \\
\hline
\end{tabular}

Another (and probably more reliable) indicator of page quality is average page-reading time. Table 2 compares time spent reading (TSR) for pages with and without annotation. For comparison, we looked at the median TSR over all the pages, by category, for all students. The median was chosen, in order to give less significance to extreme TSRs. The data shows that students spend significantly more time reading pages with annotations than those without annotations. Thus, annotation-based navigation support does indeed guide students to important pages. 
Table 2 -Effect of annotation on Time Spent Reading (TSR in second) a page

\begin{tabular}{|l|l|l|c|c|}
\hline & \multicolumn{2}{|c|}{ Median TSR } & \multicolumn{2}{c|}{ p-value } \\
\hline & Spring 2004 & Fall 2004 & Spring 2004 & Fall 2004 \\
\hline Annotated & 363 & 177 & \multirow{2}{*}{0.00001} & 0.00001 \\
\hline Not Annotated & 28 & 27 & & \\
\hline
\end{tabular}

The effect of annotation on group navigation behavior: Once we established that guiding students to annotated pages is meaningful, the next question is to ask whether or not the annotation-based navigation support succeeded in guiding students to these pages. To answer it, we computed the normalized access rate before and after the presence of public annotation. To normalize, we divided the number of page-visits by the number of possible days to access a page. Namely, the number of visits before annotation is divided by the number of days from the first day of using the system until the date of first public annotation and activity after annotation is divided by the number of days after the first public annotation until the last day of using the system. Fig. 6 shows that in spring and fall of 2004 , in most cases, more than $50 \%$ of the visits to a page were done after public annotation existed. The difference is statistically significant ( $\mathrm{p}$-value $=0.00001)$.
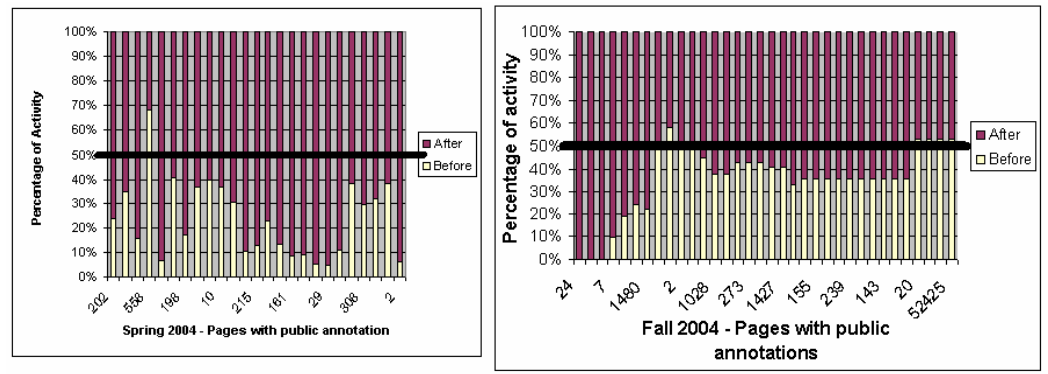

Fig. 6-Effect of presence of public annotation on student visits

Effect of personal annotation on personal navigational behavior: In the analysis of effectiveness of annotation, we looked at the effect of annotation on revisiting a page by the author of the annotation. We compared the probability of visiting a page that had been annotated by the student herself in the past with the probability of visiting a page that had not yet been annotated by this student. As shown in Table 3, in both semesters, students were more likely to revisit a page that they had already annotated. The difference in both cases is statistically significant. The students annotate pages that they want to revisit, so showing previously annotated pages is useful for navigational support.

Table 3 - Chance of self-revisit on annotated and not annotated pages

\begin{tabular}{|l|c|c|c|}
\hline & Without annotation & With annotation & p-value \\
\hline Spring 2004 & $17 \%$ & $48 \%$ & 0.01 \\
\hline Fall 2004 & $18 \%$ & $47 \%$ & 0.03 \\
\hline
\end{tabular}


Effect of thermometer on group navigation behavior: While the presence of both public and private annotations significantly influenced navigational behavior, we were not able to demonstrate the influence of the temperature icon on navigation behavior. The frequency of visits to pages with a positive temperature was not very different from pages with neutral or even negative temperatures. After more careful analysis of students' annotations we discovered that the difference between types of annotation was not very clear to the students. First, many obviously positive annotations were typed by students as being merely "general" notes. Table 4 shows that more than 50\% of "general" annotations were really "praise." Secondly, "problem" annotations were used not to indicate a bad page (as we assumed), but to report problems with the page to the teacher. In effect, the thermometer icon was useful to indicate pages with public annotations. However, the students could not rely on the "temperature" to show the quality of the information.

Table 4 - Usage of type of annotations

\begin{tabular}{|l|c|c|c|c|}
\hline & $\begin{array}{l}\text { Total Number of } \\
\text { Annotations }\end{array}$ & Praise & General & $\begin{array}{l}\text { Typed as General } \\
\text { but is really Praise }\end{array}$ \\
\hline Spring 2004 & 41 & 16 & 25 & 17 \\
\hline Fall 2004 & 51 & 24 & 21 & 11 \\
\hline
\end{tabular}

\section{Previous Work}

Several e-learning systems have been developed using the idea of social navigation. Most relevant to our project are CoFIND [6] and Educo [7]. CoFIND is a self-organized learning environment that organizes online resources through the counting of votes cast by learners. Learners can associate different types of qualities (such as "simple", "good for beginners") with each resource to help with the organization of the resources. Although CoFIND has been pretty successful among its users, it relies heavily on explicit feedback. Providing explicit feedback can interfere with the students' learning process and can increase students' extraneous cognitive load. Educo is a collaborative learning environment that supports social navigation in direct and indirect ways. Direct social navigation is supported through real-time discussion via chat rooms. Indirect social navigation is supported by annotating resources according to the number of visits. Although direct social navigation is interesting, what is more interesting and important to us is indirect social navigation. Indirect social navigation is well suited for online settings since people access the resource on an individual basis at discrete, disjointed times and locations. Direct social navigation can offer very little help to those who are not able to participate in real time discussions. It is hard to respond to discussions when people are online at different times and it is more difficult to associate topics in the discussion with specific content within the resources. The discussion could be totally irrelevant to the associated resource. In terms of indirect support of social navigation, Educo relies on 
simple implicit feedback from students: the number of visits. Although Educo also enables learners to annotate documents, this information is not used for navigation support.

\section{Conclusion}

Social navigation is a promising approach for providing navigation support inside a community of online learners. However, the challenges of collecting feedback from learners make reliable social navigation support difficult. Our results show that annotation-based social navigation support is more attractive for learners. Yet, the learners have to be motivated to annotate the tutorial pages. As a future direction of this work, we are planning to provide bridges from students' annotation to course material by letting student bookmark pages as related to specific lectures or assignments. We believe this will give more motivation and clearer navigational support to students who are authoring annotations and those who are later influenced by them.

\section{References}

1. Brusilovsky P (1996) Methods and techniques of adaptive hypermedia. User Modeling and User-Adapted Interaction 6: 87-129

2. Brusilovsky P, Chavan G, and Farzan R (2004) Social adaptive navigation support for open corpus electronic textbooks. In De Bra P and Nejdl W (eds) Third International Conference on Adaptive Hypermedia and Adaptive Web-Based Systems (AH'2004), Eindhoven, the Netherlands, August 23-26, 2004, pp. 24-33

3. Brusilovsky P and Rizzo R (2002) Using maps and landmarks for navigation between closed and open corpus hyperspace in Web-based education. New Rev Hypermedia \& Multimedia 9: 59-82

4. Dieberger A (1997) Supporting social navigation on the World Wide Web. Int J Human-Comp Interact 46: 805-825

5. Dieberger A, Dourish P, Höök K, Resnick P, and Wexelblat A (2000) Social navigation: Techniques for building more usable systems. interactions 7: 36-45

6. Dron J, Boyne C, and Mitchell R (2001) Footpaths in the stuff swamp. In Fowler W and Hasebrook J (eds) WebNet'2001, World Conference of the WWW and Internet, Orlando, FL, October 23-27, 2001, pp. 323-328

7. Kurhila J, Miettinen M, Nokelainen P, and Tirri H (2002) EDUCO - A collaborative learning environment based on social navigation. In De Bra P, Brusilovsky P and Conejo R (eds) Second International Conference on Adaptive Hypermedia and Adaptive Web-Based Systems (AH'2002), Málaga, Spain, May 29-31, 2002, pp. 242-252

8. Wexelblat A and Mayes P (1999) Footprints: History-rich tools for information foraging. In ACM Conference on Human-Computer Interaction (CHI'99), Pittsburgh, PA, pp. 270-277 